\title{
Environment-assisted quantum Minority games
}

\author{
M. Ramzan and M. K. Khan \\ Department of Physics Quaid-i-Azam University \\ Islamabad 45320, Pakistan
}

(Dated: July 30, 2021)

\begin{abstract}
The effect of entanglement and correlated noise in a four-player quantum Minority game is investigated. Different time correlated quantum memory channels are considered to analyze the Nash equilibrium payoff of the 1st player. It is seen that the Nash equilibrium payoff is substantially enhanced due to the presence of correlated noise. The behaviour of damping channels (amplitude damping and phase damping) is approximately similar. However, bitphase flip channel heavily influences the minority game as compared to other channels in the presence of correlated noise. On the other hand, phase flip channel has a symmetrical behaviour around $50 \%$ noise threshold. The significant reduction in payoffs due to decoherence is well compensated due to the presence of correlated noise. However, the Nash equilibrium of the game does not change in the presence of noise. It is seen that in case of generalized amplitude damping channel, entanglement plays a significant role at lower level of decoherence. The channel has less dominant effects on the payoff at higher values of decoherence. Furthermore, amplitude damping and generalized amplitude damping channels have almost comparable effects at lower level of decoherence $(p<0.5)$. Therefore, the game deserves careful study during its implementation due to prominent role of noise for different channels.
\end{abstract}

Keywords: Correlated noise; Minority game, entanglement; quantum channels.

\section{INTRODUCTION}

In the recent past, rapid interest has been developed in the discipline of quantum information 1] that has led to the creation of quantum game theory [2]. During last few years, number of authors have contributed to the development of quantum game theory [3-14]. Several classical games have been converted into quantum domain such as quantum prisoners' dilemma [15-31]. James et al. [32] have analyzed the quantum penny flip game using geometric algebra. Almeida et al. [33] have suggested that quantum correlations provide no advantage over classical correlations 
in a multipartite nonlocal game. Recently, Sharif and Heydari [34] have investigated Minority games for various initial states with different level of entanglement. They have shown that with the aid of entanglement and linear superposition of strategies, quantum games are shown to yield significant advantage over their classical counterparts. Some more recent investigations in the field of quantum game theory include the contributions from different authors, for details, see references [35-41].

Noise effects in different quantum games have been investigated by many authors $[5,7,10,25]$ and found interesting results. In Ref. [42], we have studied noise effects in quantum magic squares game. It is shown that the probability of success can be used to determine the characteristics of quantum channels. Implementation of decoherence and correlated noise has also been extended to the novel field of quantum information theory $[43,44]$. The Minority game has received much attention as a model of a population of agents repeatedly buying and selling in a market [45, 46]. First quantum version of a four player quantum Minority game (QMG) was examined by Benjamin and Hayden [47], and later generalized to $N$-players [48]. Flitney et al. have extended its consideration towards the implementation of decoherence [49]. Quantum channels with memory [50-52] provides a natural theoretical framework for the study of any noisy quantum communication system where correlation time is longer than the time between consecutive uses of the channel. A more general model of a quantum channel with memory was introduced by Bowen and Mancini [53] and also studied by Kretschmann and Werner [54].

In this paper, we analyze a four-player quantum Minority game influenced by different time correlated quantum memory channels, such as amplitude damping, depolarizing, bit-phase-flip and phase flip channels, parameterized by decoherence parameter $p$ and memory parameter $\mu$. Here $p \in[0,1]$ and $\mu \in[0,1]$ represent the lower and upper limits of decoherence parameter and memory parameter respectively. In addition, the generalized amplitude-damping channel, the most prominent representative of non-unital channels, is also considered parameterized by the decoherence parameter $p$ and parameter $\alpha \in[0,1]$ that depends on the temperature of the environment. It is seen that the players payoffs heavily depends on the memory of the channel under consideration. A similar behaviour of depolarizing and bit-phase flip channels is seen for maximum correlations. Amplitude damping channel influences the game more heavily as compared to the other channels. Whereas, the generalized amplitude damping channel reveals lower dissipation effects when compared with amplitude damping channel at higher level of decoherence. The Nash equilibrium payoff is substantially enhanced due to the presence of quantum memory. It is shown that memory controls the payoff reduction due to decoherence. 


\section{QUANTUM MINORITY GAMES IN A CORRELATED ENVIRONMENT}

Since noise is a major hurdle in the path of efficient information transmission from one party to the other. This noise causes a distortion of the information sent through the channel. Information transmission is said to be reliable if the probability of error, in decoding the output of the channel, vanishes asymptotically in several uses of the channel. Let the initial state of the game consists of one qubit for each player, prepared in an entangled three-qubit $G H Z$ state by an entangling operator $\hat{J}$ acting on $|0000\rangle$. In the Eisert protocol, this is achieved by applying $\hat{J}^{\dagger}$ to the game state and then making a measurement in the computational basis state. Pure quantum strategies are local unitary operators acting on a player's qubit. In a standard quantum game protocol, after the execution of players moves, the game state undergoes a positive operator valued measurement and the payoffs are determined from the classical payoff matrix, usually given for bi-matrix games. The classical pure strategies in case of Minority game are always to choose 0" or 1 . Here we use the methodolgy and notations of Ref. [49] with additional parameters $\mu$ and $\alpha$ as defined below in this section. A four-player quantum Minority game in the presence of time correlated quantum memory channels can be described using the Eisert scheme as

$$
\begin{array}{rlrl}
\rho_{0} & =\left|\Psi_{0}\right\rangle\left\langle\Psi_{0}\right| & & \text { (initial state) } \\
\rho_{1} & =\hat{J} \rho_{0} \hat{J}^{\dagger} & \text { (entanglement) } \\
\rho_{2}=D\left(\rho_{1}, p_{1}, \mu_{1}\right) & \text { (partial decoherence and correlations) } \\
\rho_{3}=\bigotimes_{k=1}^{4} \hat{M}_{k} \rho_{2} \underset{k=1}{\left.\otimes_{k=1}^{4} \hat{M}_{k}\right)^{\dagger}} & \text { (players' moves) } \\
\rho_{4}=D\left(\rho_{3}, p_{2}, \mu_{2}\right) & \text { (partial decoherence and correlations) } \\
\rho_{5}=\hat{J}^{\dagger} \rho_{4} \hat{J} & \text { (preparation for measurement) }
\end{array}
$$

to produce the final state $\rho_{f} \equiv \rho_{5}$ upon which a measurement is taken. Here $\hat{M}_{k}$ represents the $k^{\text {th }}$ player move. However, for the sake of simplicity, $p_{1}=p_{2}=p$ and $\mu_{1}=\mu_{2}=\mu$ are used in rest of the calculations. The function $D(\rho, p, \mu)$ represents a completely positive map which can be completely described in Kraus operator formalism as studied by Macchiavello and Palma [39] a Pauli channel with partial memory. The two qubit Kraus operators for such a channel can be written as

$$
A_{i j}=\sqrt{p_{i}\left[(1-\mu) p_{j}+\mu \delta_{i j}\right]} \sigma_{i} \otimes \sigma_{j}
$$

where $\sigma_{i}\left(\sigma_{j}\right)$ are usual Pauli matrices, $p_{i}\left(p_{j}\right)$ represent the quantum noise and indices $i$ and $j$ runs from 0 to 3 . The above expression means that with probability $\mu$ the channel acts on the second 
qubit with the same error operator as on the first qubit, and with probability $(1-\mu)$, it acts on the second qubit independently. Physically the parameter $\mu$ is determined by the relaxation time of the channel when a qubit passes through it. In order to remove correlations, one can wait until the channel has relaxed to its original state before sending the next qubit, however this lowers the rate of information transfer. The action of a Pauli channel with memory on $n$-qubits can be generalized in Kraus operator form as

$$
A_{i_{1} \ldots . i_{n}}=\sqrt{p_{i_{n}} \prod_{m=1}^{n-1}\left[(1-\mu) p_{i_{m}}+\mu \delta_{i_{m}, i_{m+1}}\right]} \sigma_{i_{1}} \otimes \ldots . \otimes \sigma_{i_{n}}
$$

As stated above, with probability $(1-\mu)$ the noise is uncorrelated and can be completely specified by the Kraus operators

$$
A_{i j}^{u}=\sqrt{p_{i} p_{j}} \sigma_{i} \otimes \sigma_{j}
$$

and with probability $\mu$ the noise is correlated (i.e. the channel has memory) which can be specified by the Kraus operators

$$
A_{k k}^{c}=\sqrt{p_{k}} \sigma_{k} \otimes \sigma_{k}
$$

A detailed list of single qubit Kraus operators for different quantum channels with uncorrelated noise is given in table 1 . The action of such a channel if $n$ qubits are streamed through it, can be described in operator sum representation as [5]

$$
\rho_{f}=\sum_{k_{1}, \ldots, k_{n}=0}^{n-1}\left(A_{k_{n}} \otimes \ldots . . A_{k_{1}}\right) \rho_{i n}\left(A_{k_{1}}^{\dagger} \otimes \ldots . . A_{k_{n}}^{\dagger}\right)
$$

where $\rho_{i n}$ represents the initial density matrix for quantum state and $A_{k_{n}}$ are the Kraus operators which satisfy the completeness relation

$$
\sum_{k_{n}=0}^{n-1} A_{k_{n}}^{\dagger} A_{k_{n}}=1
$$

The Kraus operators for time correlated quantum amplitude damping channel are given by Yeo and Skeen [40]

$$
A_{00}^{c}=\left[\begin{array}{llll}
\cos \chi & 0 & 0 & 0 \\
0 & 1 & 0 & 0 \\
0 & 0 & 1 & 0 \\
0 & 0 & 0 & 1
\end{array}\right], \quad A_{11}^{c}=\left[\begin{array}{llll}
0 & 0 & 0 & 0 \\
0 & 0 & 0 & 0 \\
0 & 0 & 0 & 0 \\
\sin \chi & 0 & 0 & 0
\end{array}\right]
$$


where, $0 \leq \chi \leq \pi / 2$ and is related to the quantum noise parameter as

$$
\sin \chi=\sqrt{p}
$$

The action of such a non-unital channel can be written as

$$
\Phi(\rho)=(1-\mu) \sum_{i, j=0}^{1} A_{i j}^{u} \rho A_{i j}^{u \dagger}+\mu \sum_{k=0}^{1} A_{k k}^{c} \rho A_{k k}^{c \dagger}
$$

Amplitude damping channel describes how a two-level system approaches the equilibrium due to coupling with its environment. If the environment has a finite temperature, then such a dissipation process is described by the action of a generalized amplitude-damping (GAD) channel with parameter $\alpha$ which defines a fixed state of $A_{p, \alpha}$

$$
\rho_{\infty}=\left[\begin{array}{cc}
\alpha & 0 \\
0 & 1-\alpha
\end{array}\right]
$$

If $\alpha=0$ or $\alpha=1$, the $A_{p, \alpha}$ is simply an amplitude damping channel. Single qubit Kraus operators for GAD channel are also given in table 1.

The super-operators provide a neat way of describing the evolution of quantum states in a noisy environment. In the present scheme, the Kraus operators are of dimension $2^{4}$. They are constructed from single qubit Kraus operators by taking their tensor product over all $n^{4}$ combinations

$$
A_{k}=\underset{k_{n}}{\otimes} A_{k_{n}}
$$

where $n$ is the number of Kraus operator for a single qubit channel. The final state of the game after the action of the channel can be obtained as

$$
\rho_{f}=\Phi_{p, \mu}(|\Psi\rangle\langle\Psi|)
$$

where $\Phi_{p, \mu}$ is the super-operator realizing the quantum channel parametrized by real numbers $p$ and $\mu$. The players unitary operator is an $S U(2)$ operator which represents the pure quantum strategy and is given by

$$
\hat{M}(\theta, \delta, \beta)=\left[\begin{array}{cc}
e^{i \delta} \cos (\theta / 2) & i e^{i \beta} \sin (\theta / 2) \\
i e^{-i \beta} \sin (\theta / 2) & e^{-i \delta} \cos (\theta / 2)
\end{array}\right]
$$

where $0 \leq \theta \leq \pi$ and $\pi \leq\{\delta, \beta\} \leq-\pi$. Here $\hat{M}(0,0,0)=\hat{I}$ and $\hat{M}(\pi, 0,0)=i \hat{\sigma}_{x}$ correspond to the two classical pure strategies. Entanglement is controlled through an entangling gate as given by

$$
\hat{J}(\gamma)=\exp \left(i \frac{\gamma}{2} \sigma_{x}^{\otimes 4}\right)
$$


where the parameter $\gamma$ represents the degree of entanglement of the game and $\gamma=\pi / 2$ corresponds to maximal entanglement. It is shown by Benjamin and Hayden [36] that in a four player quantum Minority game the Nash equilibrium (NE) strategy is given by

$$
\hat{\$}_{N E}=\hat{M}\left(\frac{\pi}{2}, \frac{-\pi}{16}, \frac{\pi}{16}\right)
$$

This strategic profile $\left\{\hat{\$}_{N E}, \hat{\$}_{N E}, \hat{\$}_{N E}, \hat{\$}_{N E}\right\}$ results in an NE with an expected payoff of $1 / 4$. The expectation value of the payoff to the $k^{\text {th }}$ player can be written as

$$
\left\langle \$^{k}\right\rangle=\sum_{\xi} \hat{P}_{\xi} \rho_{f} \hat{P}_{\xi}^{\dagger} \$_{\xi}^{k}
$$

where $\hat{P}_{\xi}=|\xi\rangle\langle\xi|$ is the projector onto the computational state $|\xi\rangle$, $\$_{\xi}^{k}$ is the payoff to the $k^{\text {th }}$ player when the final state is $|\xi\rangle$ and the summation is taken over $\xi$ ranging from 1 to 4 . Let us calculate the payoff of the first player when all the players resort to their optimal strategies as given by equation (21). The expected payoff of the first player influenced by amplitude damping channel reads

$$
\begin{aligned}
\$^{\mathrm{AD}}= & \frac{1}{8} \mu p^{4}+\left[\frac{1}{8}(p-1)^{6}+\mu^{2}\left(\frac{1}{8} p^{6}-\frac{5}{8} p^{5}+\frac{5}{4} p^{4}-\frac{5}{4} p^{3}+\frac{1}{2} p^{2}\right)\right. \\
& \left.+\mu\left(-\frac{1}{8}\left(p-\frac{87}{50}\right)(p-1)^{2} p\left(\left(p-\frac{44}{25}\right) p+\frac{36}{25}\right)\right)\right] \sin (\gamma)
\end{aligned}
$$

and the expected payoff of the first player in case of phase flip channel is given by

$$
\$^{\mathrm{PF}}=\frac{1}{8}\left(\left(\begin{array}{c}
-16(\mu-1)^{3} p^{4}+32(\mu-1)^{3} p^{3} \\
-4\left(5 \mu^{3}-14 \mu^{2}+15 \mu-6\right) p^{2} \\
+4\left(\mu^{3}-2 \mu^{2}+3 \mu-2\right) p+1
\end{array}\right) \sin (\gamma)+1\right)
$$

The results for the other channels are not presented due to lengthy relations. The scripts AD, GAD, Dep, BPF and PF in figures correspond to the amplitude damping, generalized amplitude damping, depolarizing, bit-phase flip and phase flip channels respectively. Our results are consistent with Ref. [49] for the case when $\mu$ reaches 0 , as clear from figure $1(\mathrm{c})$.

\section{DISCUSSIONS}

The analytical relations for payoffs as a function of decoherence parameter $p$, memory parameter $\mu$ and entanglement parameter $\gamma$ are computed for time correlated amplitude damping, depolarizing, bit-phase flip and phase flip environments. It is seen that bit-phase flip channel heavily influences the game as compared to other memory channels. 
In figure $1(\mathrm{a}, \mathrm{b})$, the Nash equilibrium payoff is plotted as a function of the memory parameter $\mu$ for $\gamma=\pi / 2$ and $p=0.3$ and 0.7 for amplitude damping, depolarizing, bit-phase flip and phase flip channels respectively. It is seen that for lower level of decoherence, it is difficult to distinguish the effect of different channels. However, for higher level of decoherence, the effect of correlated noise on the dynamics of the game become more prominent. Therefore, it is quite easy to analyze the behaviour of different memory channels. In figure $1(\mathrm{c}, \mathrm{d})$, the Nash equilibrium payoff is plotted as a function of the decoherence parameter $p$ for $\gamma=\pi / 2 \mu=0$ and 0.5 for amplitude damping, depolarizing, bit-phase flip and phase flip channels respectively. It is seen that the behaviour of phase flip channel is symmetrical around $50 \%$ decoherence. In order to see the effect of entanglement on the dynamics of the game in the presence of decoherence and correlated noise, the Nash equilibrium payoff is plotted in figure 1 (e) as a function of the entanglement parameter $\gamma$ for $p=\mu=0.3$ for different channels. It is clear from the figure that amplitude damping channel heavily influences the player's payoff. However, depolarizing and phase flip channels overlap each other with a similar behaviour for entire range of entanglement parameter.

In figure 2, the Nash equilibrium payoff is plotted as a function of the decoherence parameter $p$ and entanglement parameter $\gamma$ for $\mu=0.5$ for amplitude damping, depolarizing, bit-phase flip and phase flip channels respectively. It is seen that even in the presence of decoherence, the payoffs are sufficiently enhanced from their classical counterparts due to the presence of correlated noise. The phase flip channel shows a symmetrical behaviour around $p=1 / 2$. Furthermore, the maximum payoff corresponds to the maximal entanglement situation i.e. $\gamma=\pi / 2$ for all the cases. It is also seen that the role of entanglement is very important during the course of the game. In figure 3 , the Nash equilibrium payoff is plotted as a function of the decoherence parameter $p$ and memory parameter $\mu$ for $\gamma=\pi / 2$ for amplitude damping, depolarizing, bit-phase flip and phase flip channels respectively. It can be inferred from the figure that the game deserves a careful study during its implementation.

In figure 4, the Nash equilibrium payoff is plotted as a function of (a) decoherence parameter $p$ and parameter $\alpha$ for $\gamma=\pi / 2$ (b) decoherence parameter $p$ and entanglement parameter $\gamma$ for $\alpha=1 / \sqrt{2}$ (c) parameter $\alpha$ and entanglement parameter $\gamma$ for $p=0.5$ (d) decoherence parameter for different values of parameter $\alpha$ for $\gamma=\pi / 2$ for the generalized amplitude damping channel. It is seen that entanglement plays a significant role at lower level of decoherence (figure 4-b). From figure 4-d, it can be seen that the GAD channel is less dominant as compared to the AD channel at higher values of decoherence. However, at lower level of decoherence, both the channels (AD and GAD) are comparable to each other, where the red curve corresponds to AD channel, $\alpha=1$ 
or 0. Furthermore, the parameter $\alpha$ has symmetrical effect on the player's payoff (figure 4-c).

\section{CONCLUSIONS}

The influence of entanglement and correlated noise on a four-player quantum Minority game is analyzed. The Nash equilibrium payoff of the first player is investigated by using different time correlated quantum memory channels. The players payoffs heavily depends on the memory of the channel. It is seen that the behaviour of amplitude damping and phase damping channels is approximately similar. It is shown that bit-phase flip channel heavily influences the minority game as compared to other channels in the presence of correlated noise. Whereas, phase flip channel has a symmetrical behaviour around 50\% decoherence. It is seen that entanglement and correlated noise play a crucial role in minority games. Therefore, the game deserves a careful study during its implementation. Furthermore, the reduction in payoffs due to decoherence is well compensated due to the presence of correlated noise. However, the Nash equilibrium of the game does not change under correlated noise. Moreover, it is seen that in case of generalized amplitude

damping channel, entanglement plays a significant role at lower level of decoherence. It has smaller damping effects on the payoff at higher values of decoherence $(p>0.5)$ as compared to the amplitude damping channel. However, amplitude damping and generalized amplitude damping channels have similar effect on the player's payoffs at lower level of decoherence $(p<0.5)$.

[1] M. A. Nielson and I. L. Chuang, Quantum computation and quantum information (Cambridge University Press 2000)

[2] D. A. Meyer, Quantum Strategies, Phys. Rev. Lett. 82 (1999) 1052.

[3] J. Eisert, M. Wilkens and M. Lewenstein, Quantum Games and Quantum Strategies, Phy. Rev. Lett. 83 (1999) 3077.

[4] L. Marinatto and T. Weber, A Quantum Approach To Static Games Of Complete Information, Phys. Lett. A 272 (2000) 291.

[5] A. P. Flitney and D. Abbott, Quantum games with decoherence, J. Phys. A: Math. Theor. 38 (2005) 449.

[6] T. Cheon and A. Iqbal, Bayesian Nash Equilibria and Bell Inequalities, J. Phys. Soc. Japan 77 (2008) 024801. 
[7] M. Ramzan et al., The effect of quantum memory on quantum games, J. Phys. A: Math. Theor. 41 (2008) 055307.

[8] M. C James, A. Iqbal and D. Abbott, N-player quantum games in an EPR setting, PLoS ONE 7 (2012) e36404.

[9] P. Frackiewicz, Quantum repeated games revisited, J. Phys. A: Math. Theor. 45 (2012) 085307.

[10] D. Schneider, A new geometrical approach to Nash equilibria organization in Eisert's quantum games, J. Phys. A: Math. Theor. 45 (2012) 085303.

[11] H. Goudarzi and S. Beyrami, Effect of uniform acceleration on multiplayer quantum game, J. Phys. A: Math. Theor. 45 (2012) 225301.

[12] A. G. M. Schmidt and Milena M. Paiva, Quantum duel revisited, J. Phys. A: Math. Theor. 45 (2012) 125304.

[13] P. Frackiewicz, Quantum information approach to normal representation of extensive games, IJQI 10 4 (2012) 1250048.

[14] W. Xia and H. Cheng-Zheng, Quantum Stackelberg Duopoly with Continuous Distributed Incomplete Information, Chin. Phys. Lett. 29 (2012) 120303.

[15] A. Iqbal, T. Cheon and D. Abbott, Probablistic analysis of three player symmetric games played using EPR settings, Phys. Lett. A 372 (2008) 6564.

[16] J. Eisert and M. Wilkens, Quantum Games, J. Mod. Opt. 47 (2000) 2543.

[17] M. Ramzan and M. K. Khan, Noise effects in a three-player Prisoner's Dilemma quantum game, J. Phys. A: Math. Theor. 41 (2008) 435302.

[18] M. Ramzan and M. K. Khan, Communication aspects of a three-player Prisoner's Dilemma quantum game, J. Phys. A: Math. Theor. 42 (2009) 025301.

[19] A. Iqbal and A. H. Toor, Evolutionarily stable strategies in quantum games, Phys. Lett. A 280 (2001) 249.

[20] A. P. Flitney and D. Abbott, Quantum version of the Monty Hall problem, Phys. Rev. A 65 (2002) 062318 .

[21] G. M. D'Ariano, et al., The Quantum Monty Hall Problem, Quant. Inf. Comp. 2 (2002) 355.

[22] A. Iqbal and A. H. Toor, Quantum mechanics gives stability to Nash equilibrium, Phys. Rev. A 65 (2002) 022036.

[23] A. Iqbal and A. H. Toor, Quantum cooperative games, Phys. Lett. A 293 (2002) 103.

[24] N. F. Johnson, Playing a quantum game with a corrupted source, Phys. Rev. A 63 (2001) 020302(R).

[25] P. Gawron and J. Sladkowski, Noise effects in quantum magic squares game, Int. J. Quant. Info. 6 (2008) 667.

[26] C. F. Lee, and N. Johnson, Exploiting randomness in quantum information processing, Phys. Lett. A 301 (2002) 343.

[27] A. P. Flitney and D. Abbott, An introduction to quantum game theory, Fluct. Noise Lett. 2 (2002) R175-87 
[28] A. P. Flitney, "Aspects of Quantum Games," PhD thesis, The University of Adelaide. (2005).

[29] S. E. Landsburg, Nash Equilibria in Quantum Games. Proceedings of the American Mathematical Society 139 (2011), 4423-4434.

[30] A. Iqbal, Studies in the Theory of Quantum Games, PhD thesis, Quaid-i-Azam University, (2004), quant-ph/0503176.

[31] A. P. Flitney and L. C. L. Hollenberg, Quantum minority game utilizing various forms of entanglement. Proc. SPIE 6802, 680209 (2007).

[32] M. C. James, et al., An Analysis of the Quantum Penny Flip Game using Geometric Algebra, J. Phys. Soc. Jpn. 78 (2009) 054801.

[33] Almeida et al., Guess your neighbour's input: a multipartite non-local game with no quantum advantage, Phys. Rev. Lett. 104 (2010) 230404.

[34] P. Sharif and H. Heydari, Strategies and payoffs in quantum minority games. AIP Conf. Proc. 1327, (2011) pp. 477-481.

[35] D. Bleh, T. Calarco and S. Montangero, Quantum Game of Life, Europhysics Lett. 97 (2012) 20012.

[36] M. Ramzan, Three-player quantum Kolkata restaurant problem under decoherence, Quant. Inf. Proc. $12(2013) 577$.

[37] C. Zu et al., Experimental demonstration of quantum gain in a zero-sum game, New J. Phys. 14 (2012) 033002 .

[38] A. M. Kowalski and A. Plastino, Disjoint states and quantum games, Phys. Scr. 87 (2013) 045007.

[39] S. Balakrishnan, R. Sankaranarayanan, Classical rules and quantum strategies in penny flip game, Quant. Inf. Process. 12 (2013) 1261.

[40] Faisal S. Khan and Simon J. D. Phoenix, Gaming the Quantum, Quant. Inf. \& Comp. 13 (2013) 1.

[41] A. G. M. Schmidt and L. da Silva, Quantum russian roulette, Physica A 392 (2013) 400.

[42] M. Ramzan and M. K. Khan, Distinguishing quantum channels via magic squares game, Quant. Info. Process. 9 (2010) 667.

[43] Z. Shadman, et al., Optimal super dense coding over noisy quantum channels, New J. Phys. 12 (2010) 073042 .

[44] M. Siomau and S. Fritzsche, Entanglement dynamics of three-qubit states in noisy channels, Eur. Phys. J. D 60 (2010) 397.

[45] D. Challet, et al., Modeling market mechanism with minority game, Physica A 276 (2000) 284.

[46] E. Moro, The Minority game: an introductory guide, in Advances in Condensed Matter and Statistical Physics, eds. E. Korutcheva and R. Cuerono, Nova Science Publishers Inc. (Hauppauge 2004).

[47] S. Benjamin and P. M. Hayden, Multiplayer quantum games, Phys. Rev. A 64 (2001) 030301(R).

[48] Q. Chen, et al., N-player quantum minority game, Phys. Lett. A 327 (2004) 98.

[49] A. P. Flitney and L. C. Hollenberg, Multiplayer quantum Minority game with decoherence, Quant. Inf. Comp. 7 (2007) 111.

[50] C. Macchiavello and G. M. Palma, Entanglement-enhanced information transmission over a quantum 
channel with correlated noise, Phys. Rev. A 65 (2002) 050301.

[51] Y. Yeo and A. Skeen, Time-correlated quantum amplitude-damping channel, Phys. Rev. A 67 (2003) 064301.

[52] V. Karimipour, et. al., Entanglement and optimal strings of qubits for memory channels, Phys. Rev. A 74 (2006) 062311.

[53] G. Bowen and S. Mancini, Quantum channels with a finite memory, Phys. Rev. A 69 (2004) 01236.

[54] D. Kretschmann and R. F. Werner, Quantum Channels with Memory, Phys. Rev. A 72 (2005) 062323. 


\section{Figures captions}

Figure 1. (Color online). The Nash equilibrium payoff is plotted as a function of the memory parameter $\mu$ for $\gamma=\pi / 2$ and $(\mathrm{a}, \mathrm{b}) p=0.3$ and 0.7 , as a function of the decoherence parameter $p$ for $\gamma=\pi / 2$ and (c, d) $\mu=0$ and 0.5 , as a function of the entanglement parameter $\gamma(\mathrm{e})$ for $p=\mu=0.3$ for amplitude damping, depolarizing, bit-phase flip and phase flip channels respectively.

Figure 2. (Color online). The Nash equilibrium payoff is plotted as a function of the decoherence parameter $p$ and entanglement parameter $\gamma$ for $\mu=0.5$ for amplitude damping, depolarizing, bit-phase flip and phase flip channels respectively.

Figure 3. (Color online). The Nash equilibrium payoff is plotted as a function of the decoherence parameter $p$ and memory parameter $\mu$ for $\gamma=\pi / 2$ for amplitude damping, depolarizing, bit-phase flip and phase flip channels respectively.

Figure 4. (Color online). The Nash equilibrium payoff is plotted as a function of the decoherence parameter $p$, parameters $\alpha$ and $\gamma$ for different situations as labeled on subgraphs (a-d) for generalized amplitude damping channel.

\section{Table Caption}

Table 1. Single qubit Kraus operators for amplitude damping, generalized amplitude damping, depolarizing, bit-phase flip, bit flip and phase flip channels where $p$ represents the decoherence parameter and parameter $\alpha \in[0,1]$ corresponds to the environment with non-zero temperature (only in case of GAD channel). 
(a) $p=0.3$

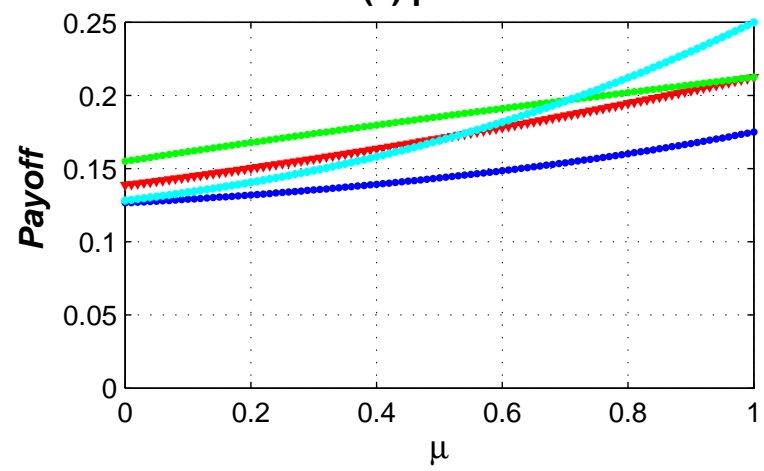

(c) $\mu=0$

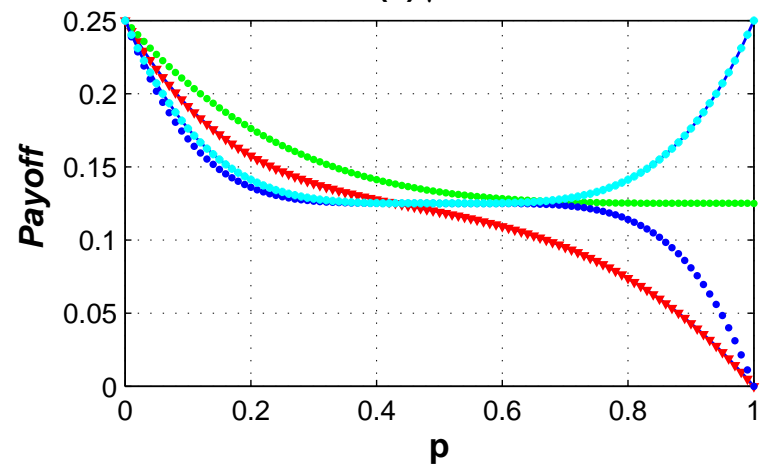

(e) $\mu=p=0.3$

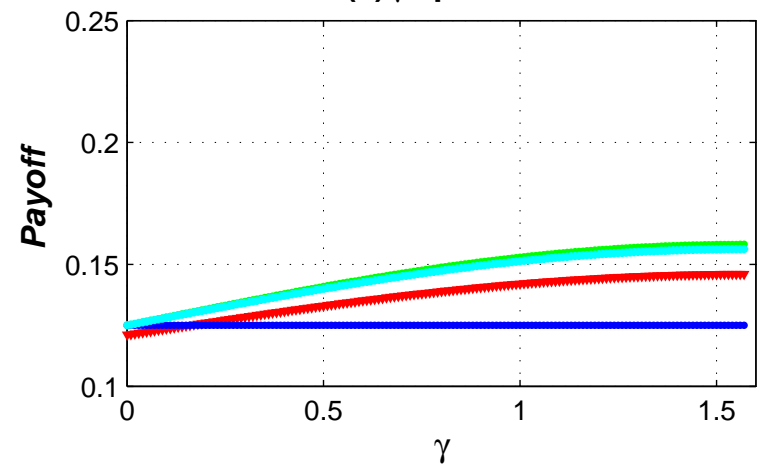

(b) $p=0.7$

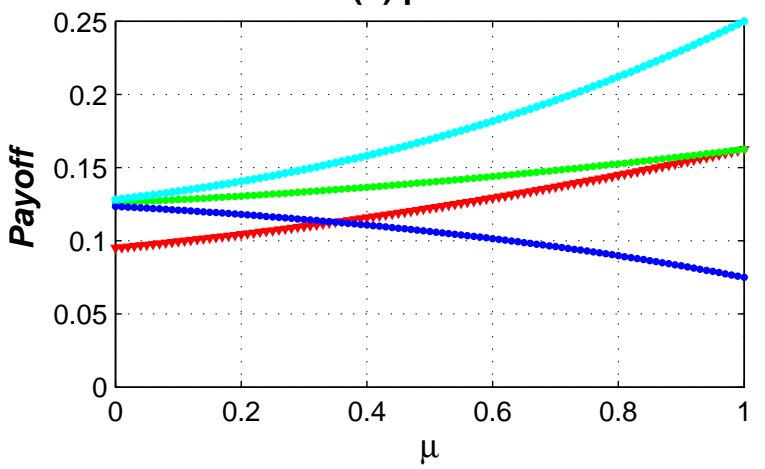

(d) $\mu=0.5$

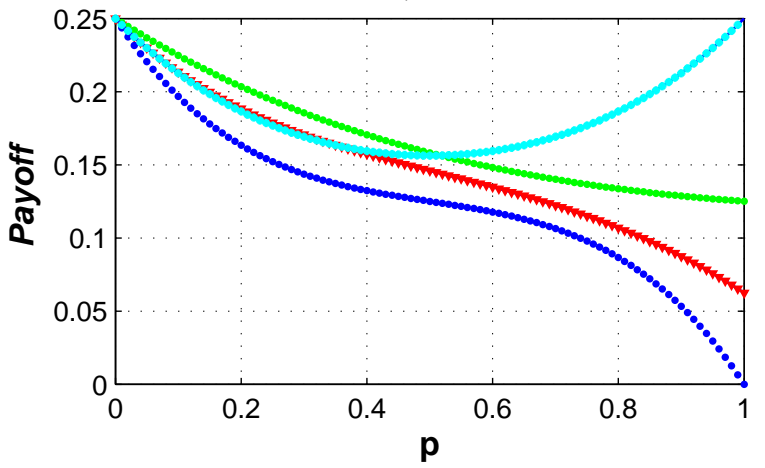

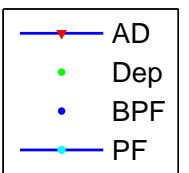

FIG. 1: (Color online). The Nash equilibrium payoff is plotted as a function of the memory parameter $\mu$ for $\gamma=\pi / 2$ and (a,b) $p=0.3$ and 0.7 , as a function of the decoherence parameter $p$ for $\gamma=\pi / 2$ and (c, d) $\mu=0$ and 0.5 , as a function of the entanglement parameter $\gamma$ (e) for $p=\mu=0.3$ for amplitude damping, depolarizing, bit-phase flip and phase flip channels respectively. 

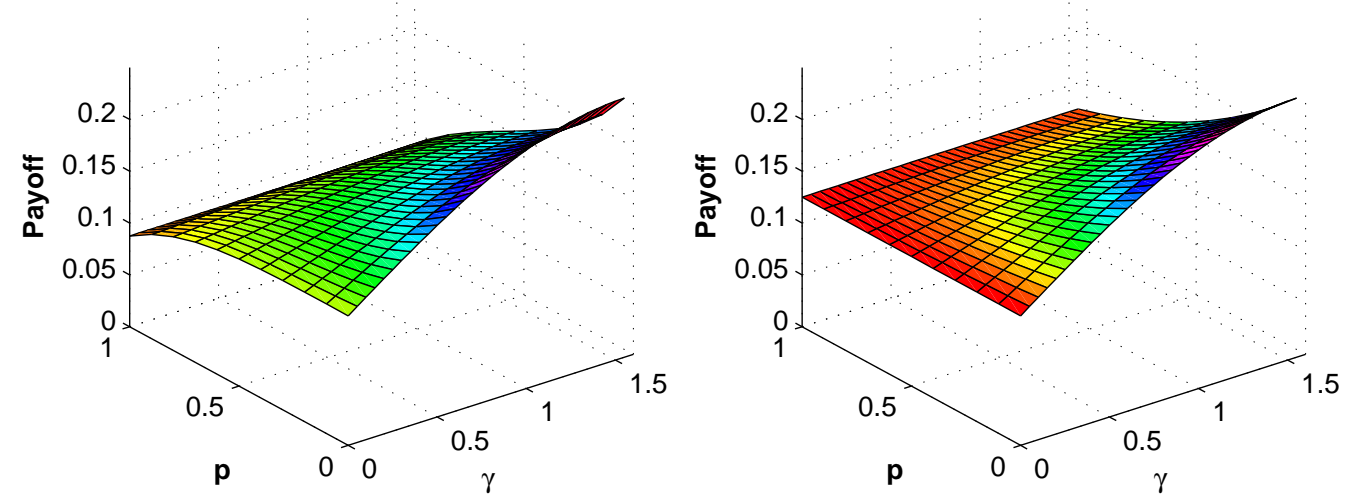

(c) Bit-phase flip
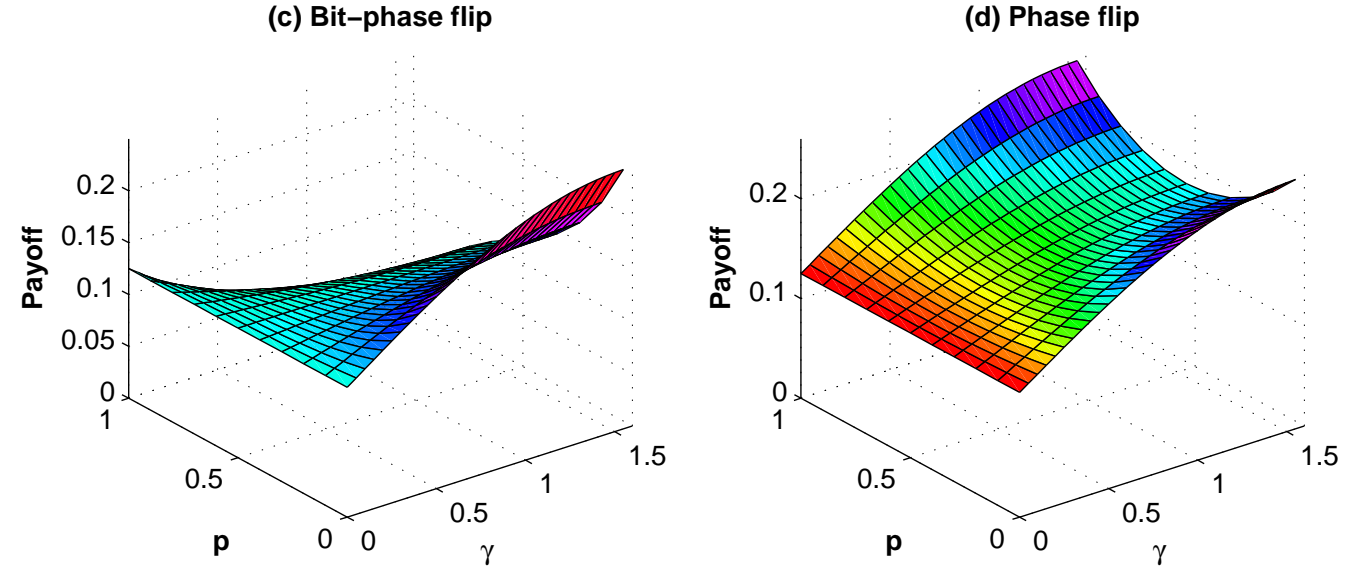

FIG. 2: (Color online). The Nash equilibrium payoff is plotted as a function of the decoherence parameter $p$ and entanglement parameter $\gamma$ for $\mu=0.5$ for amplitude damping, depolarizing, bit-phase flip and phase flip channels respectively. 
(a) Amplitude damping

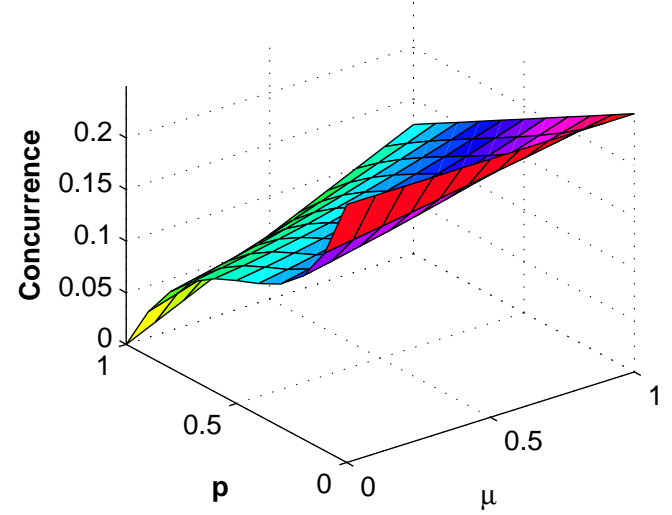

(c) Bit-phase flip

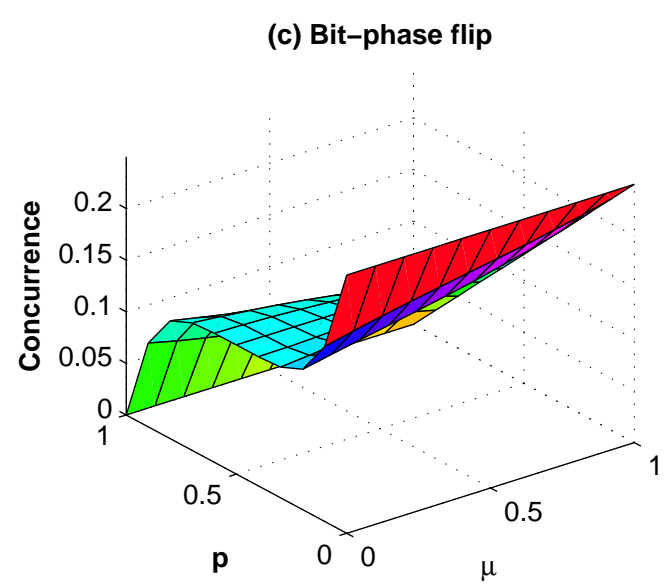

(b) Depolarizing
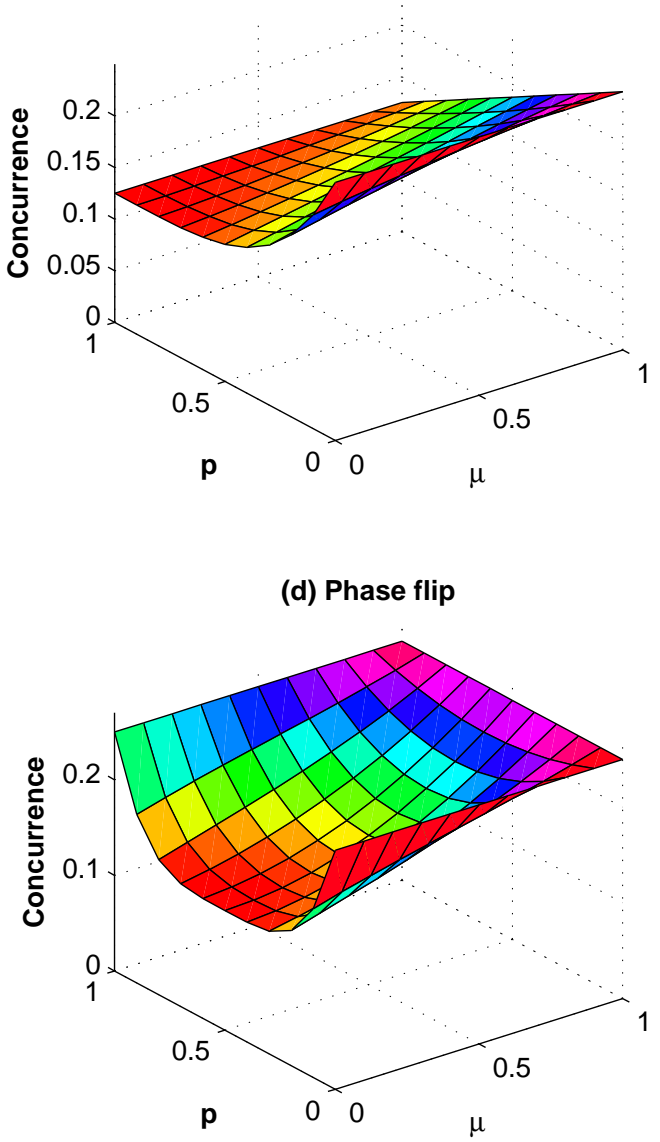

FIG. 3: (Color online). The Nash equilibrium payoff is plotted as a function of the decoherence parameter $p$ and memory parameter $\mu$ for $\gamma=\pi / 2$ for amplitude damping, depolarizing, bit-phase flip and phase flip channels respectively. 

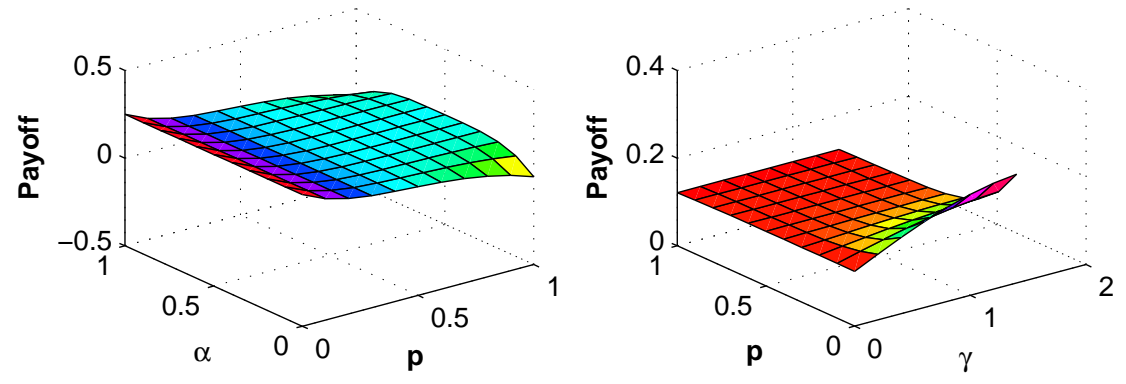

(c) $p=0.5$
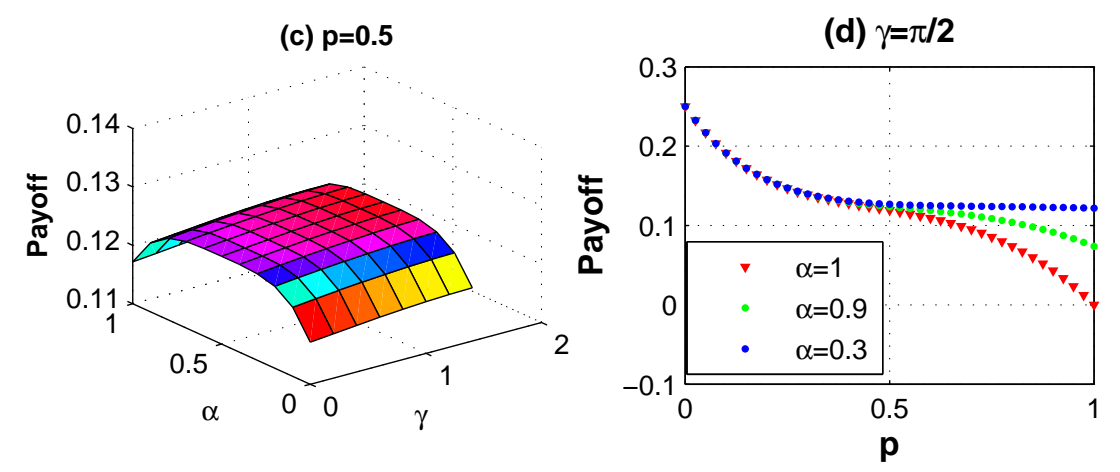

FIG. 4: (Color online). The Nash equilibrium payoff is plotted as a function of the decoherence parameter $p$, parameters $\alpha$ and $\gamma$ for different situations as labeled on subgraphs (a-d) for generalized amplitude damping channel.

TABLE I: Single qubit Kraus operators for amplitude damping, generalized amplitude damping, depolarizing, bit-phase flip, bit flip and phase flip channels where $p$ represents the decoherence parameter and parameter

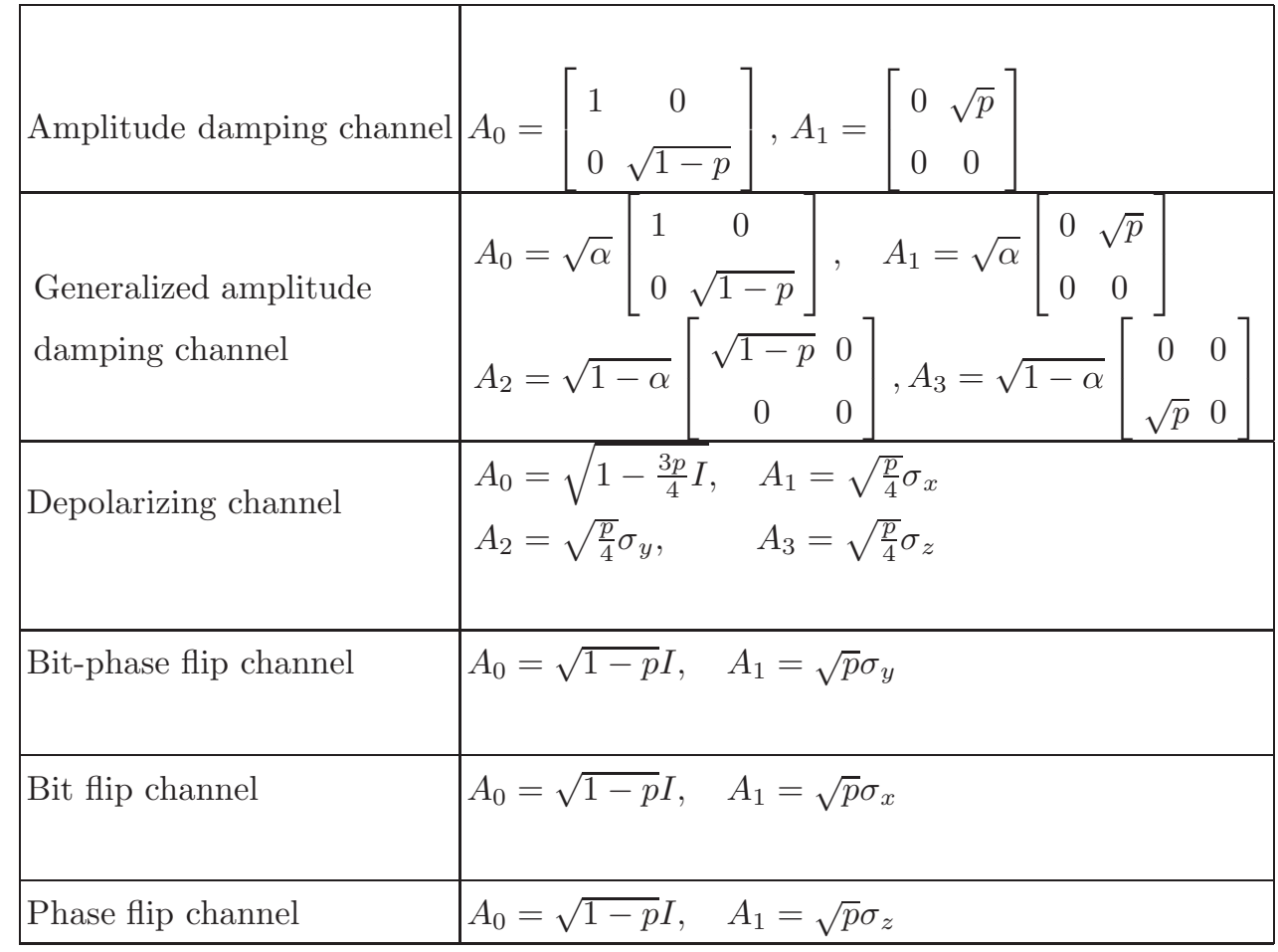

